\title{
Diffuse large B cell lymphoma (DLBCL) presenting as a spindle cell tumor of the liver: a case report
}

\author{
Bryn Haws • Da Zhang • Ivan Damjanov • \\ Jameson Forster $\cdot$ Wei Cui
}

Received: 5 September 2012 / Accepted: 4 December 2012 /Published online: 5 January 2013

(C) Springer-Verlag Berlin Heidelberg 2013

\begin{abstract}
Diffuse large B cell lymphoma (DLBCL) may present rarely as a spindle cell tumor. Only one previous case of spindle cell DLBCL involving the liver has been reported. We present a case of primary hepatic DLBCL with spindle cell features in a 67-year-old man who initially presented with elevated liver enzymes and an indistinct liver mass identified by computed tomography. The lesion was diagnosed by needle biopsy as an inflammatory pseudotumor at an outside hospital and repeat imaging suggested that the lesion had decreased in size. Thus, no treatment was given. Seven months later, he presented with jaundice and additional porta hepatic mass and lymphadenopathy. A surgical resection was performed. Microscopically, the liver mass, bile duct mass, and lymph nodes demonstrated a spindle cell infiltration interspersed with small and large lymphocytes, and eosinophils in a fibrotic background. One lymph node showed focal architectural effacement by large atypical lymphoid cells. Immunohistochemical staining of the lymph node and liver mass revealed that the tumor cells were positive for CD20, BCL-6, CD79a, and PAX-5. Proliferation index (Ki-67) ranged from 70 to $90 \%$. We concluded that both the liver and lymph nodes were involved by spindle cell DLBCL. This case is presented to underscore the importance of including DLBCL in the differential diagnosis of spindle cell lesions, especially those found in unusual locations such as the liver.
\end{abstract}

B. Haws $\cdot$ D. Zhang $\cdot$ I. Damjanov $\cdot$ W. Cui $(\bowtie)$

Department of Pathology and Laboratory Medicine,

The University of Kansans Medical Center,

3901 Rainbow Blvd,

Kansas City, KS 66160, USA

e-mail: wcui@kumc.edu

J. Forster

Department of Surgery, The University of Kansas Medical Center,

Kansas City, KS, USA
Keywords Diffuse large B cell lymphoma · Inflammatory pseudotumor $\cdot$ Spindle cell

\section{Introduction}

Diffuse large B cell lymphoma (DLBCL) has many morphologic presentations. One morphologic variant, DLBCL with spindle cell features, is extremely rare [1]. In the majority of cases reported, this variant has been described in the skin, nasal-ocular mucosa, and soft tissues [2, 3]. To the best of our knowledge, only one previous report of DLBCL with spindle cell features has been reported in the liver. In that case, the initial diagnosis was suspicious for sarcomatoid hepatocellular carcinoma [3]. Because of its rarity and spindle cell morphology, DLBCL with spindle cell features is often misdiagnosed. A high index of suspicion and utilization of immunohistochemistry is essential in establishing the diagnosis. We present a case of DLBCL with spindle cell features involving the liver, initially diagnosed as inflammatory pseudotumor.

\section{Clinical history}

A previously healthy 67 -year-old man was found to have elevated liver enzymes on routine blood work. Imaging revealed an area of abnormal decreased attenuation involving the posterior medial aspect of the right lobe of the liver measuring approximately $8 \mathrm{~cm}$ in greatest dimension (Fig. 1). A core needle biopsy of the liver lesion was performed at an outside hospital (OSH), and a diagnosis of an inflammatory pseudotumor of the liver was made. The diagnosis was confirmed by a second outside institution. Over the next 5 months, the patient remained in good health and serial computed tomography (CT) reported the liver lesion to be 


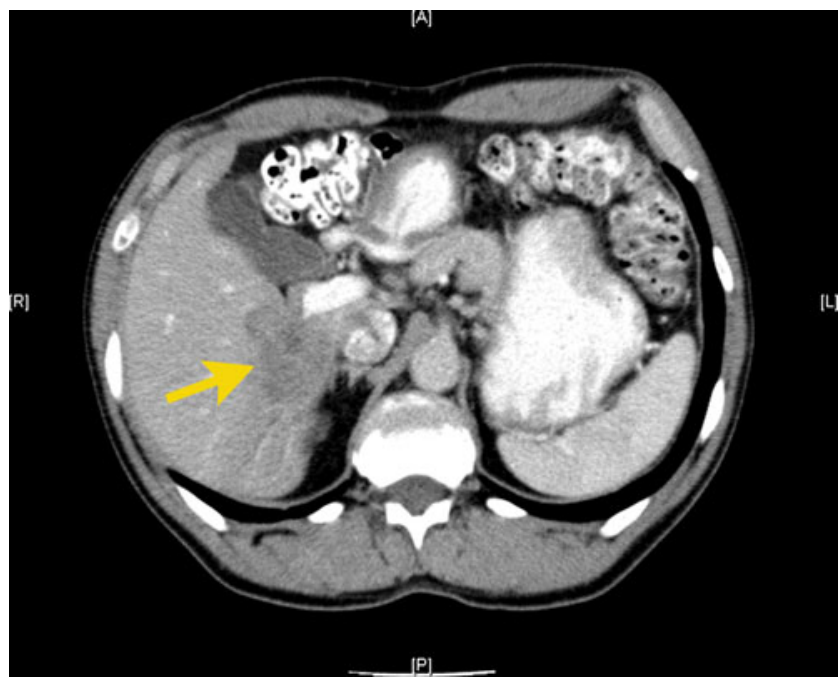

Fig. 1 CT imaging of the liver with a focal area of decreased attenuation involving the posterior medial aspect of the right lobe (arrow)

decreasing in size. Thus, no treatment was given. However, 7 months following the initial diagnosis, the patient presented to an OSH with jaundice. CT of the abdomen reported a new porta hepatic mass impinging on the biliary tree and adjacent enlarged lymph nodes. The size of the liver lesion remained unchanged. The patient was transferred to our institution for further evaluation and treatment. 18[F]fluorodeoxyglucosepositron emission tomography (PET) CT scan revealed increased metabolic activity in the right lobe liver lesion and adjacent lymph nodes at the porta hepatis. Fine needle aspiration of the lymph nodes revealed atypical epithelial cells. Surgery was consulted and a decision was made to proceed with surgical resection that would be both therapeutic and diagnostic. An exploratory laparotomy including resection of the liver mass and portal lymphadenectomy was performed.

\section{Material and methods}

The specimen was received in fresh. A portion was the specimen was sampled sterilely and submitted for flow cytometry. The specimen was sampled and tissue blocks were processed using $10 \%$ buffered neutral formalin. The specimen fixed for approximately $6 \mathrm{~h}$ prior to processing with the Tissue Tech VIP 5 processor using a 12-h processing run time.

Immunohistochemical studies on representative sections of lymph nodes and liver mass were performed at the University of Kansas Medical Center. Immunohistochemical stains performed on the lymph nodes included CD3, CD5, CD10, CD20, CD21, CD35, CD68, CD163, BCL-6, cyclin D1, MUM-1, and S100. Immunohistochemical stains performed on the liver mass included CD20, CD21, CD34, CD35, CD79a, CD117, BCL-6, PAX-5, S-100, HHF-35, ALK-1, pan-cytokeratin, desmin, smooth muscle actin, Ki-
67, hepatitis B surface antigen, and hepatitis C. Formalinfixed, paraffin-embedded tissue sections $(4-5 \mu \mathrm{m})$ were performed on either DAKO stainer using DAKO Flex Detection System (Carpinteria, California) or VentanaBenchMark XT using iView/DAB detection system (Tucson, Arizona) with commercially available antibodies according to standard manufacturing protocols. All negative controls were run with patient tissue treated with antibody diluent as the primary. Positive controls demonstrated appropriate immunolabeling. A tissue paraffin block was submitted to Mayo Clinic (Rochester, MN) and immunoglobulin gene rearrangement studies for immunoglobulin heavy chain and kappa light chain genes were performed.

In situ hybridization study for Epstein-Bar Virus (EBV)Encoded RNA (EBER) was performed on VentanaBenchMark XT using iView/Blue detection system with commercially available probe according to standard manufacturing protocols. All negative and positive controls demonstrated appropriate labeling.

\section{Results}

Grossly, the liver resection contained a $6.7 \times 4.5 \times 3.6-\mathrm{cm}$ fairly well-circumscribed mass with a pushing border and a tan-pink fibrous cut surface. Received with the liver was a gallbladder with attached hepatic duct and four lymph nodes. The cystic duct was surrounded by a $3.1 \times 3.0 \times 2.8$ $\mathrm{cm}$ lobular mass with a fibrous cut surface. A total of 13 lymph nodes were identified.

Histologically, the liver mass was composed of a spindle cell proliferation with a mixed inflammatory infiltrate of small and large lymphocytes and eosinophils in a fibrotic background (Fig. 2). No plasma cells were seen. Frequent mitotic figures were identified. The spindle cells had irregularly shaped nuclear contours with vesicular and hyperchromatic nuclei. Microscopic examination of lobular mass at the cystic duct and two of the 13 lymph nodes revealed a spindle cell proliferation identical to the process in the liver. However, in the third lymph node, there was a focal area of architectural effacement by a diffuse process composed of large cells with round to oval irregular nuclear contours, vesicular nuclei, some with prominent nucleoli, and abundant cytoplasm (Fig. 3).

Immunohistochemical stains performed on the lymph node showed that these neoplastic cells were positive for CD20 and BCL-6 (Fig. 3) and negative for CD3, CD5, CD10, CD21, CD35, CD68, CD163, cyclin D1, MUM-1, and S-100 (data not shown). A Ki-67 stain showed an 80$90 \%$ proliferation index (Fig. 3). Immunohistochemical stains performed on representative sections of the liver mass demonstrated that the spindle cells were positive for CD20, CD79a, BCL-6, and vimentin with focal and weak positivity for PAX-5 (Fig. 2). The spindle cells were negative for 
Fig. 2 Sections from the liver reveal a spindle cell proliferation in a fibrotic background with a mixed inflammatory infiltrate composed of lymphocytes and eosinophils. Hematoxylin$\operatorname{eosin}(\mathbf{a}-\mathbf{b}), \times 100$ and $\times 400$; CD20 staining (c) $\times \times 400$; CD79a staining $(\mathbf{d}), \times 400$; BCL-6 staining (e), $\times 400$; Ki-67 staining (f), $\times 400$
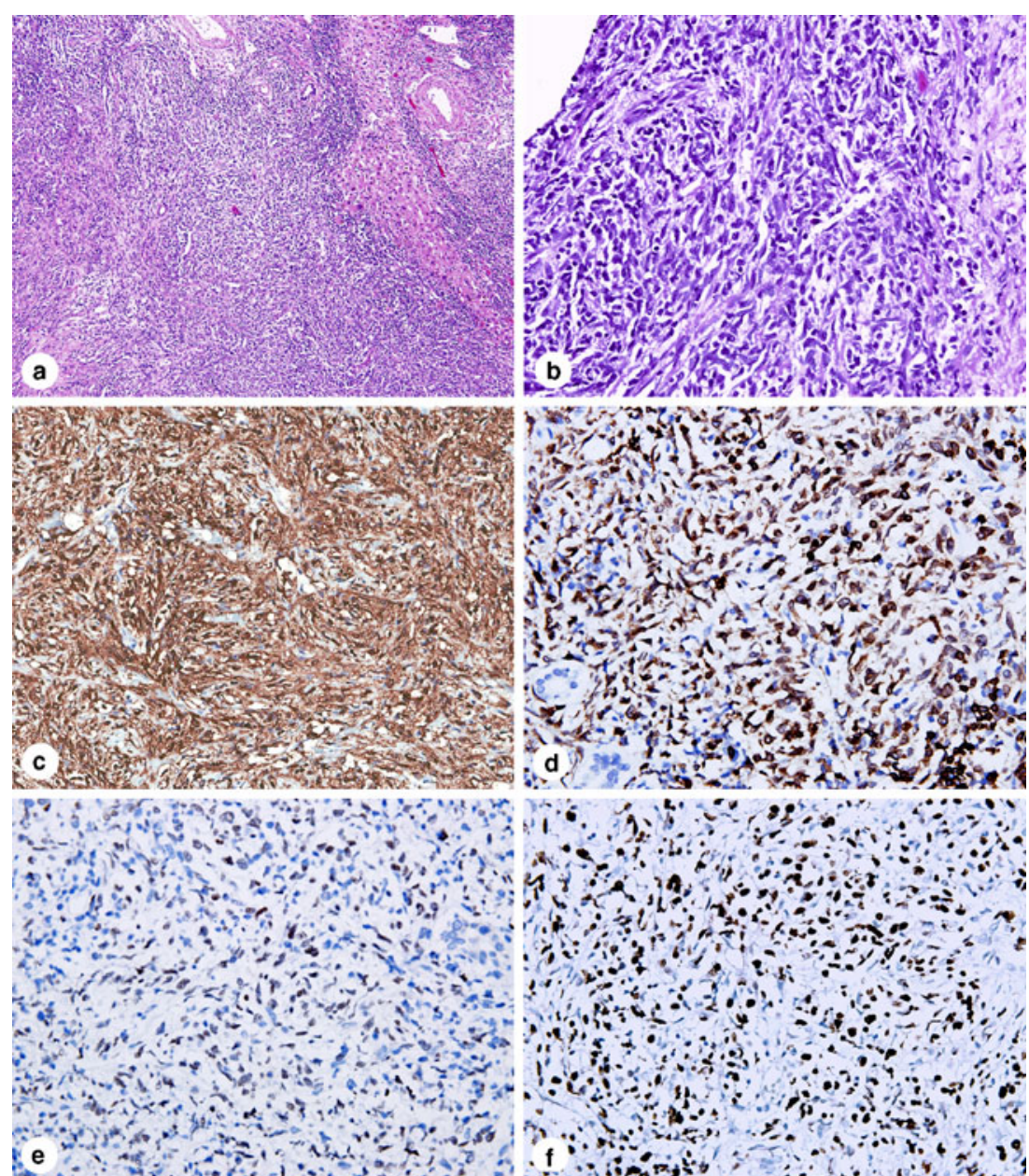

smooth muscle actin, desmin, HHF-35, CD21, CD35, CD117, CD34, S-100, pan-cytokeratin, and ALK-1 (data not shown). A Ki-67 stain showed a $70-80 \%$ proliferation index (Fig. 2). Additionally, immunostains for hepatitis B surface antigen and hepatitis $\mathrm{C}$ were negative on lymphoma and surrounding hepatocytes. EBV-ISH was negative (data not shown). Flow cytometry was reported as a negative immunophenotypic study with very few B cells present. Based on the morphologic and immunophenotypic findings, a diagnosis of a diffuse large $\mathrm{B}$ cell lymphoma with spindle cell features was made. To confirm this diagnosis, molecular studies of B cell immunoglobulin gene rearrangement performed subsequently on submitted tissue block from the liver mass at the Mayo Clinic demonstrated clonal immunoglobulin gene arrangement (data not shown).

\section{Discussion}

Rare cases of diffuse large B cell lymphoma with prominent spindle cell components have been reported. Most of these cases are reported in the skin, nasal-ocular mucosa, and soft tissue $[2,3]$. To the best of our knowledge, only one previous case of this variant of DLBCL has been reported in the liver [3]. Primary hepatic B cell lymphoma is recently defined as an extranodal lymphoma arising in the liver, with the bulk of disease localized to this site [4]. Contiguous lymph node and perihepatic tissue involvement can be seen in primary hepatic B cell lymphoma [5]. In our patient, the bulk of the disease is localized to the liver with extension to cystic duct and several lymph nodes at porta hepatis. PET CT scan fails to show increased metabolic activity in any other lymph nodes, and bone marrow biopsy performed at outside hospital is negative for lymphomatous involvement. These findings indicate that this lymphoma is a primary hepatic lymphoma.

The differential diagnosis of spindle cell lesions involving liver is broad, which includes inflammatory pseudotumor/inflammatory myofibroblastic tumor, spindle cell carcinoma, sarcoma, metastatic gastrointestinal stromal tumor (GIST), melanoma, and lymphoma. Often, DLBCL with prominent spindle cell features is initially misdiagnosed. In one study by Wang et al., the initial diagnoses of five case reports of DLBCL with prominent spindle cell features included sarcoma, carcinoma, large cell lymphoma, and inflammatory pseudotumor [2]. Immunohistochemistry is instrumental in establishing the diagnosis. In our case, the 
Fig. 3 Sections from the porta hepatic lymph node demonstrate a focal involvement by sheets of large cells with round to oval nuclei, vesicular chromatin, and abundant cytoplasm. Hematoxylin-eosin (a-b), $\times 100$ and $\times 400$; CD3 staining (c), $\times 400$; CD20 staining (d), $\times 400$; BCL-6 staining (e), $\times 400$; Ki-67 staining (f) $\times 400$

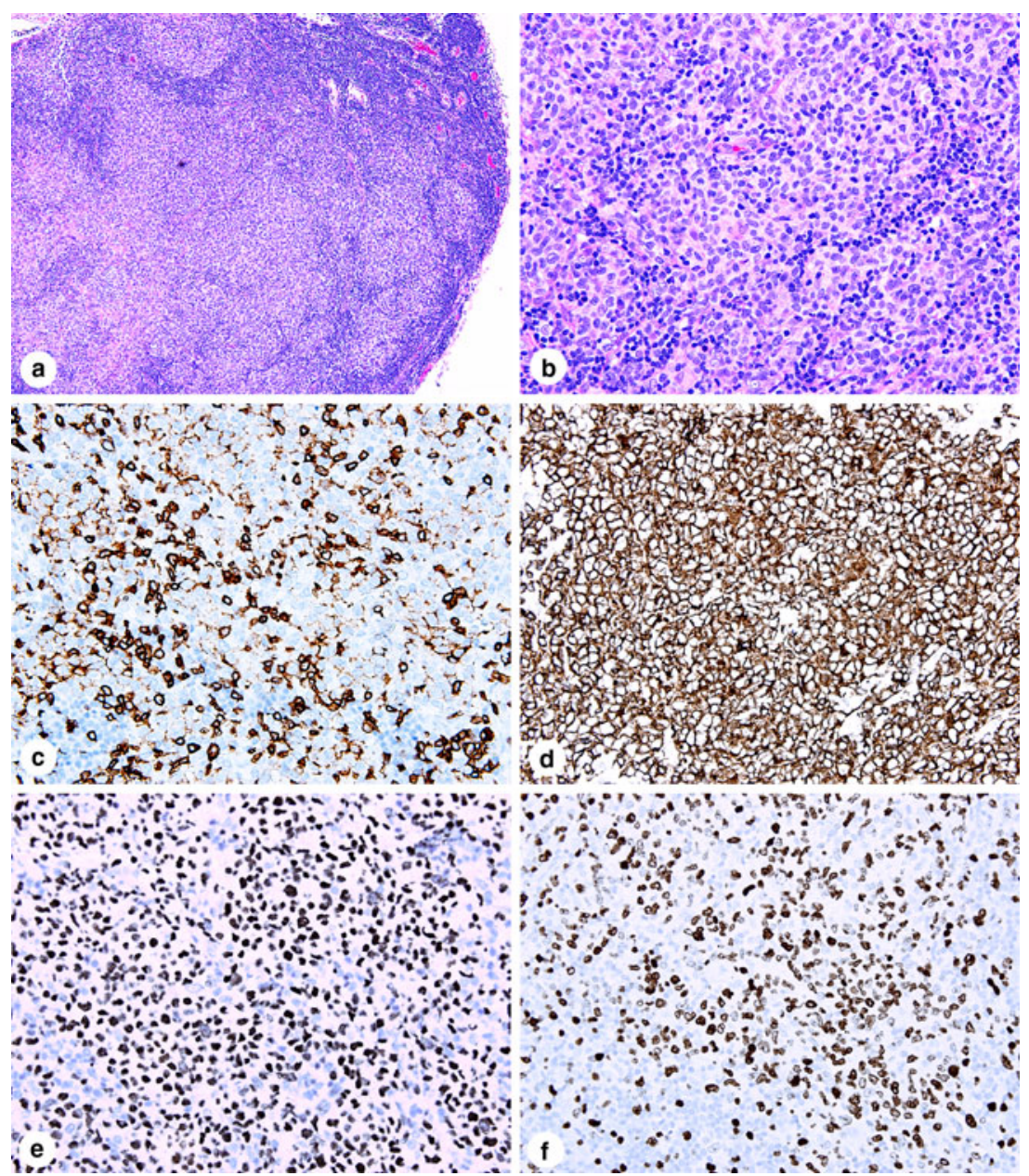

spindle cells were admixed with lymphocytes and eosinophils, making an inflammatory pseudotumor the leading initial diagnosis. As the patient was followed, the clinical behavior of the tumor warranted further workup and resection of the liver mass and lymph nodes. Morphologically, the lesion in the liver resembled inflammatory pseudotumor; however, the morphologic features of cellular atypia and hyperchromasia, frequent mitotic figures, absence of plasma cells, negativity for smooth muscle actin, and clinical aggressive behavior, including involvement of multiple lymph nodes and the cystic duct, prompted the consideration of an alternative diagnosis. Immunohistochemical staining with CD20, CD79a, as well as BCL-6 was striking, with the majority of spindle cells showing strong positivity. Inflammatory pseudotumor is just one of many neoplasms with a similar morphology to spindle cell lymphoma. Additional immunostains are needed to narrow the differential. In this case, immunohistochemical stains were used to rule out several entities including follicular dendritic cell tumor (CD21 and CD35), melanoma (S-100), spindle cell carcinoma (pan-CK), angiosarcoma (CD34), leiomyosarcoma (desmin, SMA and HHF-35), GIST (CD117), and lastly inflammatory pseudotumor/inflammatory myofibroblastic tumor (SMA, HHF-35 and ALK-1).
Lymphoma cells have been reported to assume a spindle configuration as they infiltrate bone and soft tissues $[6,7]$. They may also be spindled in the absence of stromal influence, presumably as a result of cytoskeletal aberrations [7]. SMA positivity has been reported in association with the spindle cell morphology $[2,8]$. Hayashi et al. noted two types of proliferating spindle cells in their case of DLBCL of the liver; SMA negative lymphoma cells (CD20 and CD79a positive) and SMA positive nonneoplastic spindle cells likely representing proliferating myofibroblasts (CD20 and CD79a negative) [3]. In this case, the majority of spindle cells were strongly and diffusely positive for CD20 and CD79a with no immunoreactivity to SMA. While, the significance of SMA positivity reported in a few cases is not fully understood, in the current case, its relationship with the spindle cell morphology is not observed. A recent study demonstrated that expression of fibroblast growth factor 2 (FGF2) and transforming growth factor- $\beta 1$ (TGF $\beta 1$ ) were significantly increased in the stromal cells in DLBCLs with spindle morphology in comparison to DLBCLs with conventional morphology [9]. These findings suggest that stromal alterations mediated by FGF 2 and TGF $\beta 1$ may play a role in the spindle-shaped morphology. 
Attempts have been made in order to clarify the clinical and biological features of spindle cell DLBCL. A study by Carbone et al. suggests that the genotypic and phenotypic markers of spindle cell DLBCL are consistent with germinal center B cell origin [10]. Their study also noted that the germinal center phenotype was associated with primary extranodal origin, normal lactate dehydrogenase levels, and good response to treatment. In this case, lymphoma cells are positive for BCL-6 and negative for CD10 and MUM; therefore, these findings are consistent with germinal center B cell origin.

In summary, we describe a case of primary hepatic DLBCL with spindle cell features. The spindle cells were found to be of germinal center B cell origin, a finding consistent with previously reported cases [10]. As is common in most of spindle cell DLBCL, this case was initially misdiagnosed. This case underscores the importance of including lymphoma in the differential diagnosis of a spindle cell lesions and its consideration even in unusual locations such as the liver.

\section{References}

1. Cerroni L, El-Shabrawi-Caelen L, Fink-Puches R et al (2000) Cutaneous spindle-cell B-cell lymphoma: a morphologic variant of cutaneous large B-cell lymphoma. Am J Dermatopathol 22:299-304

2. Wang J, Sun NC, Nozawa Y et al (2001) Histological and immunohistochemical characterization of extranodal diffuse large-cell lymphomas with prominent spindle cell features. Histopathology 39:476-481

3. Hayashi H, Ohtani H, Ueda T et al (2006) Primary hepatic lymphoma with spindle cell components: a case report. Virchows Arch 449:591-596

4. Bosman FT, Carneiro F, Hruban RH et al (2010) WHO classification of tumours of the digestive system. IARC, Lyon, pp 239-240

5. Kikuma K, Watanabe J, Oshiro Y et al (2012) Etiological factors in primary hepatic B-cell lymphoma. Virchows Arch 460:379-387

6. Chan JK, Buchanan R, Fletcher CD (1990) Sarcomatoid variant of anaplastic large-cell Ki-1 lymphoma. Am J Surg Pathol 14:983988

7. Nozawa Y, Wang J, Weiss LM et al (2001) Diffuse large B-cell lymphoma with spindle cell features. Histopathology 38:177178

8. Fung CH, Antar S, Yonan T et al (1993) Actin-positive spindle cell lymphoma. Arch Pathol Lab Med 117:1053-1055

9. Kimura Y, Arakawa F, Kiyasu J et al (2012) A spindle cell variant of diffuse large B-cell lymphoma is characterized by T-cell/myofibrohistio-rich stromal alterations: analysis of 10 cases and a review of the literature. Eur J Haematol 89:302-310

10. Carbone A, Gloghini A, Libra M et al (2006) A spindle cell variant of diffuse large B-cell lymphoma possesses genotypic and phenotypic markers characteristic of a germinal center B-cell origin. Mod Pathol 19:299-306 